\title{
On The Weight Distribution of Fixed-Rate Raptor Codes
}

\author{
Francisco Lázaro*, Enrico Paolini ${ }^{\dagger}$, Gianluigi Liva*, Gerhard Bauch ${ }^{\ddagger}$ \\ ${ }^{*}$ Institute of Communications and Navigation of DLR (German Aerospace Center), \\ Wessling, Germany. Email: \{Francisco.LazaroBlasco,Gianluigi.Liva\}@dlr.de \\ ${ }^{\dagger}$ Department of Electrical, Electronic, and Information Engineering, University of Bologna, \\ Cesena, Italy. Email: e.paolini@unibo.it \\ ${ }_{\ddagger}^{\ddagger}$ Institute for Telecommunication, Hamburg University of Technology \\ Hamburg, Germany. Email: Bauch@tuhh.de
}

\begin{abstract}
In this paper Raptor code ensembles with linear random precodes in a fixed-rate setting are considered. An expression for the average distance spectrum is derived and this expression is used to obtain the asymptotic exponent of the weight distribution. The asymptotic growth rate analysis is then exploited to develop a necessary and sufficient condition under which the fixed-rate Raptor code ensemble exhibits a strictly positive typical minimum distance.
\end{abstract}

\section{INTRODUCTION}

Fountain codes [1] are erasure codes potentially able to generate an endless amount of encoded symbols. As such, they find application in contexts where the channel erasure rate is not a priori known. The first class of practical fountain codes, Luby Transform (LT) codes, was introduced in [2] together with an iterative belief propagation (BP) decoding algorithm that is efficient when the number of input symbols $k$ is large. One of the shortcomings of LT codes is that in order to have a low probability of unsuccessful decoding, the encoding cost per output symbol has to be $\mathcal{O}(\ln (k))$. Raptor codes [3] overcome this problem. They consist of a serial concatenation of an outer precode $\mathcal{C}$ with an inner LT code. The LT code design can thus be relaxed requiring only the recovery of a fraction $1-\gamma$ of the input symbols with $\gamma$ small. This can be achieved with linear encoding complexity. The outer precode is responsible for recovering the remaining fraction of input symbols, $\gamma$. If the precode $\mathcal{C}$ is linear-time encodable, then the Raptor code has a linear encoding complexity, $\mathcal{O}(k)$, and, therefore, the overall encoding cost per output symbol is constant with respect to $k$. Furthermore, Raptor codes are universally capacity-achieving on the binary erasure channel.

Most of the works on LT and Raptor codes consider BP decoding which has a good performance for very large input blocks ( $k$ at least in the order of a few tens of thousands symbols). Often, in practice smaller blocks are used. For example, for the Raptor codes standardized in [4] and [5] the recommended values of $k$ range from 1024 to 8192 . For these input block lengths, the performance under BP decoding degrades considerably. In this context, an efficient maximum likelihood (ML) decoding algorithm in the form of inactivation decoding [6] may be used in place of BP. Recently, ML decoding for Raptor and LT codes has been analyzed [7]-[9], focusing however mainly on their decoding complexity under inactivation decoding.

Despite their rateless capability, Raptor codes represent an excellent solution for fixed-rate communication schemes requiring powerful erasure correction capabilities with low decoding complexity. Hence, it is not surprising that Raptor codes are actually used in a fixed-rate setting by existing communication systems (see e.g. [10]). In this context, the performance under ML erasure decoding is determined by the distance properties of the fixed-rate Raptor code ensemble, that to the best knowledge of the authors have not yet been analyzed.

In this paper we analyze the distance properties of fixedrate Raptor codes. In particular, we focus on the case where the precode is picked from the linear random ensemble. The choice of this ensemble is not arbitrary. The precode used by some standardized Raptor codes [4], [5] is a concatenation of two systematic codes, the first being a high-rate regular low density parity check (LDPC) code and the second being pseudo-random code characterized a dense parity check matrix. These precodes were designed to behave like codes of the linear random ensemble in terms of rank properties, but allowing a fast algorithm for matrix vector multiplication [11]. Thus, we conjecture that the results obtained for the ensemble considered in this work may give (as a first approximation) hints on the distance properties of Raptor codes employed in existing systems. For this Raptor code ensemble we develop a necessary and sufficient condition to guarantee a strictly positive typical minimum distance. The condition is found to depend on the degree distribution of the inner LT code and on the code rates of both the inner LT code and the (outer) precode. A necessary condition is also derived which, beyond the inner/outer code rates, depends on the average output degree only.

The rest of the paper is organized as follows. The main definitions are introduced in Section II. Section III provides the derivation of the average weight distribution of this Raptor code ensemble and of the associated growth rate. Section IV provides necessary and sufficient conditions for a positive typical minimum distance. The conclusions follow in Section V. 


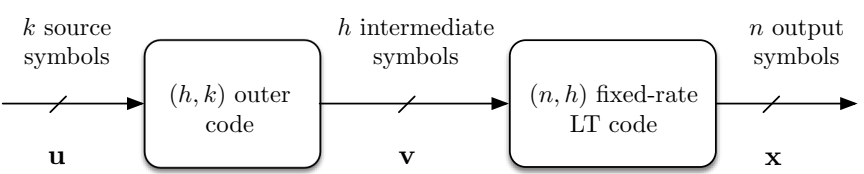

Fig. 1. Raptor codes consist of a serial concatenation of a linear block code (pre-code) with a LT code.

\section{PRELIMINARIES}

We consider fixed-rate Raptor code ensembles based on the encoder structure depicted in Figure 1. The encoder is given by a serial concatenation of an $(h, k)$ outer precode with an $(n, h)$ inner fixed-rate LT code. We denote by $\mathbf{u}$ the outer encoder input, and by $\mathbf{U}$ the corresponding random vector. Similarly, $\mathbf{v}$ and $\mathbf{x}$ denote the input and the output of the fixed-rate LT encoder, with $\mathbf{V}$ and $\mathbf{X}$ being the corresponding random vectors. The vectors $\mathbf{u}, \mathbf{v}$ and $\mathbf{x}$ are composed by $k$, $h$ and $n$ symbols each. The symbols of $\mathbf{u}$ are referred to as source symbols, whereas the symbols of $\mathbf{v}$ and $\mathbf{x}$ are referred to as intermediate and output symbols, respectively.

We restrict to symbols belonging to $\mathbb{F}_{2}$. We denote by $w_{\mathrm{H}}(\mathbf{a})$ the Hamming weight of a binary vector a. For a generic LT code output symbol $x_{i}, \operatorname{deg}\left(x_{i}\right)$ denotes the output symbol degree, i.e., the number of intermediate symbols that are added (in $\mathbb{F}_{2}$ ) to produce $x_{i}$. We will respectively denote by $r_{\mathrm{o}}=$ $k / h, r_{\mathrm{i}}=h / n$, and $r=k / n=r_{\mathrm{o}} r_{\mathrm{i}}$ the rates of the outer code, the inner LT code, and the Raptor code.

We consider the ensemble of Raptor codes $\mathscr{C}\left(\mathscr{C}_{\mathrm{o}}, \Omega, r_{\mathrm{i}}, r_{\mathrm{o}}, n\right)$ obtained by a serial concatenation of an outer code in the $\left(r_{\mathrm{i}} n, r_{\mathrm{o}} r_{\mathrm{i}} n\right)$ binary linear random block code ensemble, $\mathscr{C}_{\mathrm{O}}$, with all possible realizations of an $\left(n, r_{\mathrm{i}} n\right)$ fixed-rate LT code with output degree distribution $\Omega=\left\{\Omega_{1}, \Omega_{2}, \Omega_{3}, \ldots, \Omega_{d_{\max }}\right\}$, where $\Omega_{i}$ corresponds to the probability of having an output symbol of degree $i$. We finally denote as $\bar{\Omega}$ the average output degree, $\bar{\Omega}=\sum_{i} i \Omega_{i}$.

In the following we make use of the notion of exponential equivalence [12], as follows. Two real-valued positive sequences $a(n)$ and $b(n)$ are said to be exponentially equivalent, writing $a(n) \doteq b(n)$, when

$$
\lim _{n \rightarrow \infty} \frac{1}{n} \log _{2} \frac{a(n)}{b(n)}=0
$$

Moreover, given two pairs of reals $\left(x_{1}, y_{1}\right)$ and $\left(x_{2}, y_{2}\right)$, we write $\left(x_{1}, y_{1}\right) \succeq\left(x_{2}, y_{2}\right)$ when $x_{1} \geq x_{2}$ and $y_{1} \geq y_{2}$.

\section{Distance Spectrum of Fixed-Rate Raptor Code ENSEMBLES}

\section{A. Average Weight Enumerator}

Let us denote by $A_{w}$ the average weight enumerator (WE) of the ensemble $\mathscr{C}\left(\mathscr{C}_{\mathrm{o}}, \Omega, r_{\mathrm{i}}, r_{\mathrm{o}}, n\right)$. For $w>0$ we have

$$
A_{w}=\sum_{l=1}^{h} \frac{A_{l}^{\circ} A_{l, w}^{\mathrm{i}}}{\left(\begin{array}{c}
h \\
l
\end{array}\right)}
$$

where $A_{l}^{\circ}$ is the average WE of the outer precode, and $A_{l, w}^{\mathrm{i}}$ is the average input output weight enumerator (IOWE) of the inner LT code. The average WE of an $(h, k)$ random code is known to be (see [13])

$$
A_{l}^{\circ}=\left(\begin{array}{l}
h \\
l
\end{array}\right) 2^{-h\left(1-r_{\mathrm{o}}\right)} \text {. }
$$

We now focus on the average IOWE of the LT code. Let us denote by $l$ the Hamming weight of the input of the LT encoder, and let us assume that the output symbol of the LT code has degree $j$. Let us denote by $p_{j, l}$ the probability that any of the $n$ output bits of the LT encoder takes the value 1 given that the Hamming weight of the intermediate word is $l$ and the degree of the LT code output symbol is $j$, i.e.,

$$
p_{j, l}:=\operatorname{Pr}\left\{X_{i}=1 \mid w_{\mathrm{H}}(\mathbf{V})=l, \operatorname{deg}\left(X_{i}\right)=j\right\}
$$

for any $i \in\{1, \ldots, n\}$. This probability may be expressed as

$$
p_{j, l}=\sum_{\substack{i=\max (1, l+j-h) \\
i \text { odd }}}^{\min (l, j)} \frac{\left(\begin{array}{l}
j \\
i
\end{array}\right)\left(\begin{array}{c}
h-j \\
l-i
\end{array}\right)}{\left(\begin{array}{c}
h \\
l
\end{array}\right)} .
$$

Removing the conditioning on $j$ we obtain $p_{l}$, the probability of any of the $n$ output bits of the LT encoder taking value 1 given a Hamming weight $l$ for the intermediate word, i.e.,

$$
p_{l}:=\operatorname{Pr}\left\{X_{i}=1 \mid w_{\mathrm{H}}(\mathbf{V})=l\right\}
$$

for any $i \in\{1, \ldots, n\}$. We have

$$
p_{l}=\sum_{j=1}^{d_{\max }} \Omega_{j} p_{j, l}
$$

Since the output bits are generated by the LT encoder independently of each other, the Hamming weight of the LT codeword conditioned to an intermediate word of weight $l$ is a binomially distributed random variable with parameters $n$ and $p_{l}$. Hence, we have

$$
\operatorname{Pr}\left\{w_{\mathrm{H}}(\mathbf{X})=w \mid w_{\mathrm{H}}(\mathbf{V})=l\right\}=\left(\begin{array}{c}
n \\
w
\end{array}\right) p_{l}^{w}\left(1-p_{l}\right)^{n-w} .
$$

The average IOWE of a LT code may be now easily calculated multiplying (5) by the number of weight- $l$ intermediate words, yielding

$$
A_{l, w}^{\mathrm{i}}=\left(\begin{array}{l}
h \\
l
\end{array}\right)\left(\begin{array}{l}
n \\
w
\end{array}\right) p_{l}^{w}\left(1-p_{l}\right)^{n-w} .
$$

Making use of (2), (3) and (6), for $w>0$ the average WE of the fixed-rate Raptor code ensemble can be expressed as

$$
A_{w}=\left(\begin{array}{l}
n \\
w
\end{array}\right) 2^{-h\left(1-r_{\mathrm{o}}\right)} \sum_{l=1}^{h}\left(\begin{array}{l}
h \\
l
\end{array}\right) p_{l}^{w}\left(1-p_{l}\right)^{n-w}
$$

\section{B. Growth Rate of Fixed-Rate Raptor Code Ensembles}

In this subsection we compute the asymptotic exponent (growth rate) of the weight distribution for the ensemble $\mathscr{C}_{\infty}\left(\mathscr{C}_{\mathrm{o}}, \Omega, r_{\mathrm{i}}, r_{\mathrm{o}}\right)$, that is the ensemble $\mathscr{C}\left(\mathscr{C}_{\mathrm{o}}, \Omega, r_{\mathrm{i}}, r_{\mathrm{o}}, n\right)$ in the limit where $n$ tends to infinity for constant $r_{\mathrm{i}}$ and $r_{\mathrm{o}}$. Hereafter, we denote the normalized output weight of the Raptor encoder by $\tilde{w}=w / n$ and the normalized output weight of the precoder (input weight to the LT encoder) by $\tilde{l}=l / h$. 


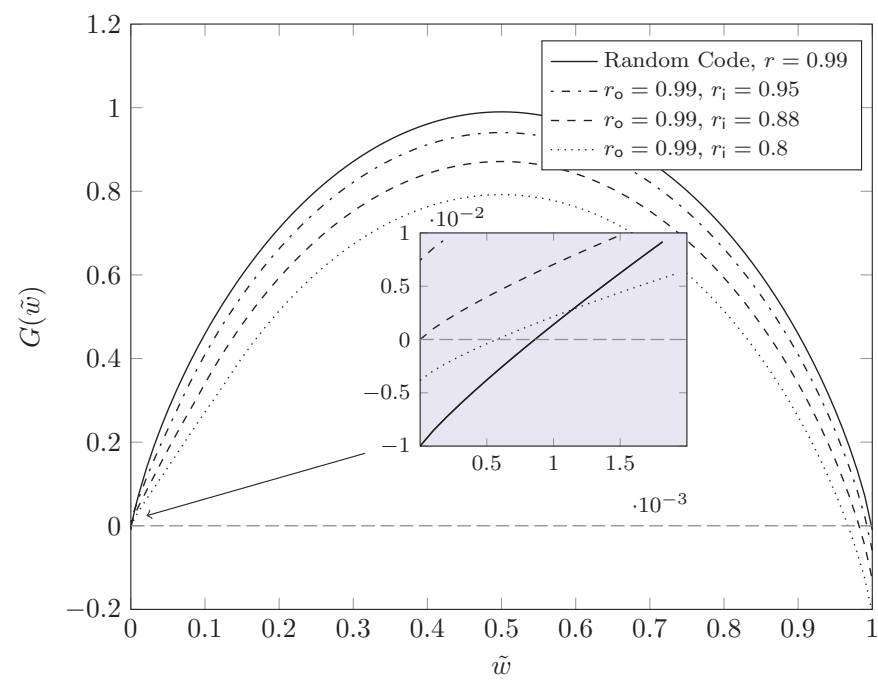

Fig. 2. Growth rate vs. normalized output weight $\tilde{w}$. The solid line shows the growth rate of a linear random code with rate $r=0.99$. The dotdashed, dashed, and dotted lines show the growth rates $G(\tilde{w})$ of the ensemble $\mathscr{C}_{\infty}\left(\mathscr{C}_{\mathrm{o}}, \Omega^{(2)}, r_{\mathrm{i}}, r_{\mathrm{o}}=0.99\right)$ for $r_{\mathrm{i}}=0.95,0.88$ and 0.8 , respectively.

Using the well-known exponential equivalence $\left(\begin{array}{c}n \\ n \tilde{w}\end{array}\right) \doteq 2^{n \mathrm{H}_{\mathrm{b}}(\tilde{w})}$, where $\mathrm{H}_{\mathrm{b}}$ is the binary entropy function, for large $n$ the multiplicative term in front of the summation in (7) fulfills

$$
\left(\begin{array}{l}
n \\
w
\end{array}\right) 2^{-h\left(1-r_{\mathrm{o}}\right)} \doteq 2^{n\left[\mathrm{H}_{\mathrm{b}}(\tilde{w})-r_{\mathrm{i}}\left(1-r_{\mathrm{o}}\right)\right]}:=\beta .
$$

Therefore, $A_{w}=A_{\tilde{w} n}$ in (7) fulfills

$$
A_{\tilde{w} n} \doteq \beta \sum_{\tilde{l}} 2^{n\left[r_{\mathrm{i}} \mathrm{H}_{\mathrm{b}}(\tilde{l})+\tilde{w} \log _{2} p_{\tilde{l}}+(1-\tilde{w}) \log _{2}\left(1-p_{\tilde{l}}\right)\right]}
$$

where

$$
p_{\tilde{l}}=\sum_{j=1}^{d_{\max }} \Omega_{j} p_{j, \tilde{l}}
$$

and

$$
p_{j, \tilde{l}}=\frac{1}{2}\left[1-(1-2 \tilde{l})^{j}\right] .
$$

Using the result

$$
\sum_{\alpha} 2^{n f(\alpha)} \doteq \max _{\alpha} 2^{n f(\alpha)}
$$

we can simplify the expression of $A_{\tilde{w} n}$ as

$$
A_{\tilde{w} n} \doteq 2^{n\left[\mathrm{H}_{\mathrm{b}}(\tilde{w})-r_{\mathrm{i}}\left(1-r_{\mathrm{o}}\right)+\mathrm{f}_{\max }(\tilde{w})\right]}
$$

where

$$
\mathrm{f}_{\max }(\tilde{w}):=\max _{\tilde{l}} \mathrm{f}(\tilde{w}, \tilde{l})
$$

and

$$
\mathrm{f}(\tilde{w}, \tilde{l}):=r_{\mathrm{i}} \mathrm{H}_{\mathrm{b}}(\tilde{l})+\tilde{w} \log _{2} p_{\tilde{l}}+(1-\tilde{w}) \log _{2}\left(1-p_{\tilde{l}}\right) .
$$

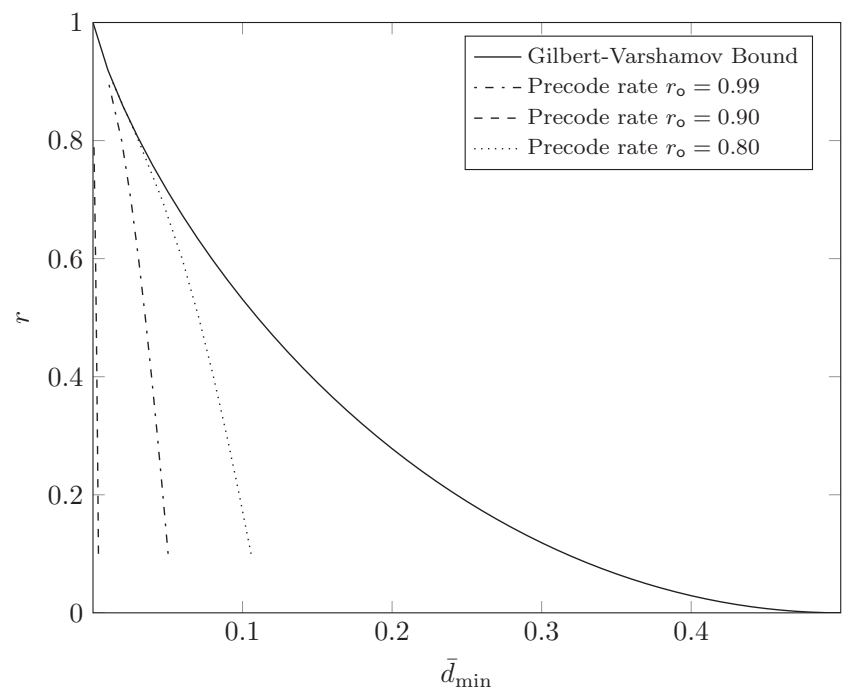

Fig. 3. Overall rate $r$ vs. the typical minimum distance $\bar{d}_{\min }$. The solid line represents the asymptotic Gilbert-Varshamov bound. The different dashed and dotted lines represent Raptor codes ensembles $\mathscr{C}_{\infty}\left(\mathscr{C}_{0}, \Omega^{(2)}, r_{\mathrm{i}}=r / r_{\mathrm{o}}, r_{\mathrm{o}}\right)$ with different outer code rates, $r_{\mathrm{o}}$.

The asymptotic exponent of the fixed-rate Raptor code ensemble weight distribution is finally

$$
\begin{aligned}
G(\tilde{w}) & :=\lim _{n \rightarrow \infty} \frac{1}{n} \log _{2} A_{\tilde{w} n} \\
& =\mathrm{H}_{\mathrm{b}}(\tilde{w})-r_{\mathrm{i}}\left(1-r_{\mathrm{o}}\right)+\mathrm{f}_{\max }(\tilde{w}) .
\end{aligned}
$$

Moreover, the real number

$$
\bar{d}_{\min }:=\inf \{\tilde{w}>0: G(\tilde{w})>0\}
$$

is the typical minimum distance of the ensemble.

Fig. 2 shows $G(\tilde{w})$ for the ensemble $\mathscr{C}_{\infty}\left(\mathscr{C}_{\mathrm{o}}, \Omega^{(2)}, r_{\mathrm{i}}, r_{\mathrm{o}}\right)$, where $\Omega^{(2)}$ is the output degree distribution used in the standards [4], [5] (see details in Table I) and $r_{\mathrm{o}}=0.99$ for three different $r_{\mathrm{i}}$ values. It can be observed how the curve for $r_{\mathrm{i}}=0.95$ does not cross the $x$-axis, the curve for $r_{\mathrm{i}}=0.88$ has $\bar{d}_{\min }=0$ and the curve for $r_{\mathrm{i}}=0.8$ has $\bar{d}_{\min }=0.0005$. The figure also shows the growth rate of the precode, a linear random code with $r=0.99$. It can be observed how the typical minimum distance of the precode is larger than that of the concatenated (Raptor) code.

Fig. 3 shows the overall rate $r$ of the Raptor code ensemble $\mathscr{C}_{\infty}\left(\mathscr{C}_{\mathrm{o}}, \Omega^{(2)}, r_{\mathrm{i}}=r / r_{\mathrm{o}}, r_{\mathrm{o}}\right)$ versus the typical minimum distance $\bar{d}_{\text {min }}$. It can be observed how, for constant overall rate $r, \bar{d}_{\min }$ increases as the outer code rate $r_{\mathrm{o}}$ decreases. It also can be observed how decreasing $r_{\mathrm{o}}$ allows to get closer to the asymptotic Gilbert-Varshamov bound.

\section{RATE REGIONS}

We are now interested in determining whether the ensemble exhibits good typical distance properties. More specifically, we are interested in the existence of a strictly positive typical minimum distance. A sufficient condition for having a positive typical minimum distance is

$$
\lim _{\tilde{w} \rightarrow 0^{+}} G(\tilde{w})<0
$$


which implies

$$
r_{\mathrm{i}}\left(1-r_{\mathrm{o}}\right)>\lim _{\tilde{w} \rightarrow 0^{+}} f_{\max }(\tilde{w}) .
$$

Unfortunately, a closed-form expression for the right-hand side of (8) does not exist in general. However, the order of limit and the maximization can be inverted by observing that the function $f_{\max }$ is right-continuous at $\tilde{w}=0$, that is

$$
\lim _{\tilde{w} \rightarrow 0^{+}} f_{\max }(\tilde{w})=f_{\max }(0) .
$$

It is now possible to recast the right-hand side of (8) as

$$
\begin{aligned}
\lim _{\tilde{w} \rightarrow 0^{+}} f_{\max }(\tilde{w}) & =\lim _{\tilde{w} \rightarrow 0^{+}} \max _{\tilde{l}} f(\tilde{w}, \tilde{l})=\max _{\tilde{l}} \lim _{\tilde{w} \rightarrow 0^{+}} f(\tilde{w}, \tilde{l}) \\
& =\max _{\tilde{l}}\left[r_{\mathrm{i}} \mathrm{H}_{\mathrm{b}}(\tilde{l})+\log _{2}\left(1-p_{\tilde{l}}\right)\right] \\
& =: \mathrm{f}_{\max }^{*}\left(r_{\mathrm{i}}\right)
\end{aligned}
$$

where we emphasized that $\mathrm{f}_{\max }^{*}$ hides a dependency on $r_{\mathrm{i}}$. Computing (9) implies carrying out a maximization which cannot generally be computed analytically. However, the function to be maximized is sufficient well behaved so that the maximization can be done numerically in an efficient manner.

Definition 1 (Positive typical minimum distance region). We define the positive typical minimum distance region of a Raptor code ensemble as the set $\mathscr{P}$ of code rate pairs $\left(r_{\mathrm{i}}, r_{\mathrm{o}}\right)$ for which the ensemble possesses a positive typical minimum distance. Formally:

$$
\mathscr{P}:=\left\{\left(r_{\mathrm{i}}, r_{\mathrm{o}}\right) \succeq(0,0) \mid \bar{d}_{\text {min }}\left(\Omega, r_{\mathrm{i}}, r_{\mathrm{o}}\right)>0\right\},
$$

Where we have used the notation $\bar{d}_{\min }=\bar{d}_{\min }\left(\Omega, r_{\mathrm{i}}, r_{\mathrm{o}}\right)$ to emphasize the dependence on $\Omega, r_{\mathrm{i}}$ and $r_{\mathrm{o}}$.

Theorem 1. An inner positive typical minimum distance region, $\mathscr{I}$, is given by

$$
\mathscr{I}:=\left\{\left(r_{\mathrm{i}}, r_{\mathrm{o}}\right) \succeq(0,0) \mid r_{\mathrm{i}}\left(1-r_{\mathrm{o}}\right)>\mathrm{f}_{\max }^{*}\left(r_{\mathrm{i}}\right)\right\} .
$$

Proof. It follows from (8) being a sufficient condition for having a positive typical minimum distance.

Theorem 2. The inner positive typical minimum distance region $\mathscr{I}$ and the positive typical minimum distance $\mathscr{P}$ region coincide, $\mathscr{I} \equiv \mathscr{P}$.

Proof. Due to space constraints we provide only a sketch of the proof. The argument is based on the observation that any pair $\left(r_{\mathrm{i}}, r_{\mathrm{o}}\right)$ such that $r_{\mathrm{i}}\left(1-r_{\mathrm{o}}\right)<\lim _{\tilde{w} \rightarrow 0^{+}} \mathrm{f}_{\max }(\tilde{w})$ cannot belong to $\mathscr{P}$ since for these pairs $\lim _{\tilde{w} \rightarrow 0^{+}} G(\tilde{w})>0$. An analysis must then be carried out for those $\left(r_{\mathrm{i}}, r_{\mathrm{o}}\right)$ pairs such that $r_{\mathrm{i}}\left(1-r_{\mathrm{o}}\right)=\lim _{\tilde{w} \rightarrow 0^{+}} \mathrm{f}_{\max }(\tilde{w})$, meaning $\lim _{\tilde{w} \rightarrow 0^{+}} G(\tilde{w})=$ 0 . For these $\left(r_{\mathrm{i}}, r_{\mathrm{o}}\right)$ pairs, the only possibility for having a positive typical minimum distance is

$$
\lim _{\tilde{w} \rightarrow 0^{+}} G^{\prime}(\tilde{w})<0 .
$$

The proof is completed by showing that the above condition never holds, regardless of $\lim _{\tilde{w} \rightarrow 0^{+}} G(\tilde{w})$ (that is regardless the considered $\left(r_{\mathrm{i}}, r_{\mathrm{o}}\right)$ pair).

Although Theorems 1 and 2 fully characterize the positive typical minimum distance $\mathscr{P}$, they require the calculation of
TABLE I

DEGREE DisTRIBUTIONS $\Omega^{(1)}$, DEFINED IN [4], [5] AND $\Omega^{(2)}$, DEFINED IN [3]

\begin{tabular}{|c|c|c|}
\hline Degree & $\Omega^{(1)}$ & $\Omega^{(2)}$ \\
\hline \hline 1 & 0.0098 & 0.0048 \\
\hline 2 & 0.4590 & 0.4965 \\
\hline 3 & 0.2110 & 0.1669 \\
\hline 4 & 0.1134 & 0.0734 \\
\hline 5 & & 0.0822 \\
\hline 8 & & 0.0575 \\
\hline 9 & & 0.0360 \\
\hline 10 & 0.1113 & \\
\hline 11 & 0.0799 & \\
\hline 18 & & 0.0012 \\
\hline 19 & & 0.0543 \\
\hline 40 & 0.0156 & \\
\hline 65 & & 0.0182 \\
\hline 66 & & 0.0091 \\
\hline \hline $\bar{\Omega}$ & 4.6314 & 5.825 \\
\hline
\end{tabular}

$\mathrm{f}_{\max }^{*}$. In the following we introduce an outer region that can be computed more easily, and only depends on the average output degree.

Proposition 1. The positive typical minimum distance region region of a fixed-rate Raptor code ensemble $\mathscr{C}_{\infty}\left(\mathscr{C}_{0}, \Omega, r_{\mathrm{i}}, r_{\mathrm{o}}\right)$ fulfills $\mathscr{P} \subseteq \mathscr{O}$, where

$$
\mathscr{O}:=\left\{\left(r_{\mathrm{i}}, r_{\mathrm{o}}\right) \succeq(0,0) \mid r_{\mathrm{i}} \leq \min \left(\phi\left(r_{\mathrm{o}}\right), \frac{1}{r_{\mathrm{o}}}\right)\right\}
$$

with

$$
\phi\left(r_{\mathrm{o}}\right)=\frac{\bar{\Omega} \log _{2} r_{\mathrm{o}}}{\mathrm{H}_{\mathrm{b}}\left(1-r_{\mathrm{o}}\right)-\left(1-r_{\mathrm{o}}\right)} .
$$

Proof. The proof goes by lower bounding $A_{\tilde{w} n}$ for $\tilde{w} \rightarrow 0^{+}$. Observing (2) we can see how $A_{\tilde{w} n}$ is summation of the number of Hamming weight $\tilde{w} n$ codewords generated by all possible input weights to the LT encoder. A lower bound to $A_{\tilde{w} n}$ is the number of Hamming weight $\tilde{w} n$ codewords generated only by inputs to the LT encoder of weight $\tilde{l}=1-r_{\mathrm{o}}$. Manipulating the expression obtained and making use of the exponential equivalency introduced in Section II the expression in (10) is obtained.

It is important to point out that the asymptotic exponent of the weight distribution captures linear-sized codewords [14]. Codewords whose weight grows with a sublinear weight should be subject to an ad-hoc analysis.

We now consider two different output degree distributions given in Table. I. The first one is the output degree distribution used in the standards [4], [5], which we will refer to as $\Omega^{(1)}$. Then, we consider a distribution $\Omega^{(2)}$ which was designed in [3] for $k=120000$.

In Fig. 4 we show the positive typical minimum distance region, $\mathscr{P}$ for $\Omega^{(1)}$ and $\Omega^{(2)}$ together with their outer bound to the positive growth region $\mathscr{O}$. It can be observed how the outer bound is tight in both cases except for inner codes rates close to $r_{\mathrm{i}}=1$. The figure also shows several isorate curves, along which the overall rate of the Raptor code $r$ stays constant. For example, in order to have a positive typical minimum distance and an overall rate $r=0.95$, the figure shows that the rate of 


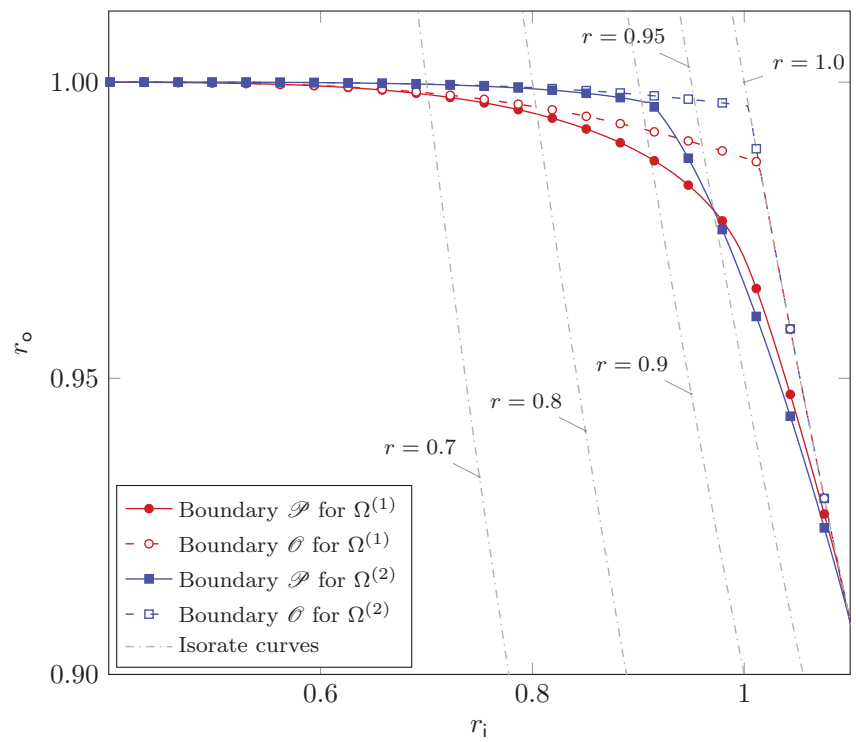

Fig. 4. Positive growth rate region. The solid and dotted lines represent the positive growth-rate region of $\Omega^{(1)}$ and $\Omega^{(2)}$ and the dashed line represents its outer bound. The markers represent the rate point at which codes in the standards [4], [5] operate for different values of $k$.

the precode must lay below $r_{\mathrm{o}}<0.978$ for both distributions. It is quite remarkable that for precode rates below $r_{\mathrm{o}}<0.978$ there exist a region in which $\Omega^{(1)}$ exhibits a positive typical minimum distance and $\Omega^{(2)}$ does not, although the average output degree of $\Omega^{(1)}$ is considerably lower than that of $\Omega^{(2)}$. This exemplifies the fact that the distance properties of a Raptor code ensemble depend not only on $r_{\mathrm{i}}$ and $r_{\mathrm{o}}$ and $\bar{\Omega}$ but also on the degree distribution $\Omega$.

\section{CONCLUSIONS}

In this work we have considered ensembles of fixed-rate Raptor codes which use linear random codes as precodes. We have derived the expression of the average weight enumerator of an ensemble and the expression of the growth rate of the weight enumerator as functions of the rate of the outer code and the rate and degree distribution of the inner LT code. Based on these expressions we are able to determine necessary and sufficient conditions to have Raptor code ensembles with a positive typical minimum distance. A simple necessary condition has been developed too, which requires (besides the inner and outer code rates) the knowledge of the average output degree only. Despite the fact that only binary Raptor codes have been considered, an extension to higher order fields is possible with a limited effort.

The work presented in this paper helps to understand the behavior of fixed-rate Raptor codes under ML decoding and it can be used to design Raptor codes with good distance properties, for example, using numerical optimization.

\section{ACKNOWLEDGEMENTS}

The authors would like to acknowledge Dr. Massimo Cicognani and Dr. Mark Flanagan for the useful discussions.

\section{REFERENCES}

[1] J. Byers, M. Luby, and M. Mitzenmacher, "A digital fountain approach to reliable distribution of bulk data," IEEE J. Select. Areas Commun., vol. 20, no. 8, pp. 1528-1540, Oct. 2002.

[2] M. Luby, "LT codes," in Proc. of the 43rd Annual IEEE Symp. on Foundations of Computer Science, Vancouver, Canada, Nov. 2002, pp. 271-282.

[3] M. Shokrollahi, "Raptor codes," IEEE Trans. Inf. Theory, vol. 52, no. 6, pp. 2551-2567, Jun. 2006.

[4] 3GPP TS 26.346 V11.1.0, "Technical specification group services and system aspects; multimedia broadcast/multicast service; protocols and codecs," Jun. 2012.

[5] M. Luby, A. Shokrollahi, M. Watson, and T. Stockhammer, "RFC 5053: Raptor forward error correction scheme: Scheme for object delivery," IETF, Tech. Rep., Oct. 2007.

[6] M. Shokrollahi, S. Lassen, and R. Karp, "Systems and processes for decoding chain reaction codes through inactivation," Feb. 2005, US Patent 6,856,263.

[7] F. Lázaro Blasco, G. Liva, and G. Bauch, "LT code design for inactivation decoding," in Proc. 2014 IEEE Inf. Theory Workshop, Hobart, Australia, Nov. 2014.

[8] _ _Enhancing the LT component of Raptor codes for inactivation decoding," in Proc. 2015 International ITG Conf. on Systems, Commun. and Coding, Hamburg, Germany, Feb. 2015.

[9] K. Mahdaviani, M. Ardakani, and C. Tellambura, "On Raptor code design for inactivation decoding," IEEE Commun. Lett., vol. 60, no. 9 , pp. 2377-2381, Sep. 2012.

[10] ETSI TR 102993 V1.1.1, "Digital Video Broadcasting (DVB); upper layer FEC for DVB systems," Feb. 2011.

[11] A. Shokrollahi and M. Luby, "Raptor codes," Found. and Trends on Commun. and Inf. Theory, vol. 6, pp. 213-322, Mar. 2009.

[12] T. M. Cover and J. A. Thomas, Elements of information theory, 2nd ed. New York: Wiley, 2006, chapter 3.

[13] R. G. Gallager, "Low-density parity-check codes," Ph.D. dissertation, Dep. Electrical Eng., M.I.T, Cambridge, MA, Jul. 1963.

[14] C. Di, R. Urbanke, and T. Richardson, "Weight distribution of lowdensity parity-check codes," IEEE Trans. Inf. Theory, vol. 52, no. 11, pp. 4839-4855, Nov. 2006. 